\title{
Nur für Schwindelfreie? Eine Geographie politischer Praktiken nach Hannah Arendt
}

\author{
Florian Dünckmann \\ Department of Geography, Christian-Albrechts-Universität, 24098 Kiel, Deutschland \\ Correspondence: Florian Dünckmann (duenckmann@geographie-uni-kiel.de)
}

Received: 21 December 2018 - Revised: 30 November 2019 - Accepted: 27 April 2020 - Published: 15 May 2020

\begin{abstract}
Kurzfassung. Times of crisis often call the legitimacy of existing social orders into question. These practices of dispute and debate that question, challenge, or affirm the rules that govern our social life are what constitutes the realm of the political. This article fathoms the potential of a Political Geography that makes political practices its main point of interest. Arendt's political philosophy provides the foundation for a geography of political practices that asks about (a) the way in which the possibility and necessity of the political is tied to the spatiality of our human condition, (b) the relation of political practices to spatial structures and their production of particular places, spaces and scales, and (c) the role which materiality plays in stabilizing, constraining and shaping political practices. Combining insights from Arendt's concept of political action with recent ideas of practice theories, a definition of political practices that relies on three characteristics - reflexivity, perspectivity, and expressivity - is introduced. I will argue that these metapragmatic practices, although they distinguish themselves from pragmatic practices, nevertheless, always remain embedded in and related to the web of our everyday doings.
\end{abstract}

\section{Einleitung}

In Zeiten der Krise wird die Gültigkeit bestehender sozialer Strukturen oft in Frage gestellt. Seien es die Krisen der Europäischen Union, der repräsentativen Demokratie oder des Kapitalismus: Krisendiskurse sind nicht nur ein zentraler Bestandteil gesellschaftlicher Selbstbeschreibung (Runkel und Everts, 2017:476), sie geben auch Anlass für eine Befragung der ihr zugrundeliegenden Regeln. Allerdings ergeben sich in Krisenzeiten aber auch Gelegenheiten, das bestehende Gesellschafts- und Herrschaftsmodell zu bestätigen und $\mathrm{zu}$ festigen, z.B. indem machtvolle Akteure nun ihre Handlungsbereitschaft und Managementfähigkeit unter Beweis stellen und damit zeigen können, dass die herrschenden Problemdefinitionen und Lösungsstrategien die richtigen sind und dass die bestehende Interpretation der sozialen Realität alternativlos ist. Kurzum, Krisen sind Zeiten der erhöhten Sensibilität für Fragen nach der Gültigkeit ansonsten unhinterfragt bleibender gesellschaftlicher Routinen und Regeln. In diesen Momenten der Unsicherheit schließen sich an die Frage „Was sollen wir jetzt tun?" tiefergehende und grundsätzlichere Fragen an, wie „Haben wir es bis jetzt rich- tiggemacht?“ oder „Wie sollen wir künftig entscheiden, was zu tun ist?".

Diese Praxis der Auseinandersetzung - bewusst oder vorbewusst, expliziert oder impliziert, kritisch oder affirmativ - mit den Regeln, die unsere gesellschaftliche Praxis leiten, bildet den Kern dessen, was im Folgenden im weitesten Sinne als Politik bzw. das Politische bezeichnet werden soll. Jüngere Ansätze der politischen Analyse definieren das Politische eher als Tätigkeit denn als eine institutionelle Architektur (z.B. Butler, 2012; Connolly, 2011; Isin und Nielsen, 2008; Woodward et al., 2012; Tully, 2008; Žižek, 1999). Politik ist verwurzelt in konkretem Tun und Sprechen; überall dort, wo Menschen ihre eigene Praxis und die ihrer Mitbürger verhandeln, machen Sie Politik. Dies geschieht nicht nur innerhalb der etablierten Institutionen politischer Meinungsbildung (z.B. Parlamente, politische Demonstrationen); auch scheinbar unpolitische Situationen, wie z.B. bei Diskussionen über mögliche Arbeitsabläufe in Unternehmen oder in grundlegenden Auseinandersetzungen in Familien oder Wohngemeinschaften, können politische Schauplätze darstellen. 
In diesem Sinne kann auch Hannah Arendt als eine Denkerin neu gelesen werden, die zu ihrer Zeit bereits auf die performative Dimension der Politik bestanden hat. Anschließend an die jüngere Rezeption von Arendts Ideen in der Geographie (siehe z.B. Barnett, 2012; Cassegård, 2014; Collins, 2010; Debarbieux, 2011; Dikeç, 2012a, b, 2013; Mustafa und Brown, 2010) möchte ich in diesem Artikel die Möglichkeiten einer Politischen Geographie ausloten, die Arendts Definition von Politik als konzeptionellen Zugangspunkt wählt und die politische Praktiken in den Mittelpunkt des Interesses rückt. Wenn Praktiken als räumliche Phänomene aufgefasst werden (vgl. Schatzki, 2015), eröffnet sich für die Politische Geographie die Frage, welche spezifischen Räume, Orte, Beziehungen und Maßstäbe politische Praktiken hervorbringen. Ein solcher Blick auf konkrete Situationen sollte dann nicht allein in der Lage sein, Praktiken des Regierens zu untersuchen (Everts et al., 2019), sondern auch subtilere politische Momente an unspektakulären Orten zu identifizieren.

Dabei soll u.a. auch die These vertreten werden, dass diese politischen Momente nicht im Gegensatz zu den Praktiken des Alltags stehen, sondern vielmehr eng mit ihnen verwoben sind. Daraus folgt allerdings nicht, dass ohnehin ,alles politisch ist': So produktiv diese These von der Allgegenwärtigkeit politisch hinterfragbarer Machtstrukturen für die Kritik verdeckter Herrschaftsmuster sein kann, so wenig eignet sie sich nach Fraser (1989) als Grundidee einer Politischen Theorie. Auf der anderen Seite ist damit aber auch keine Beschränkung auf den Bereich gemeint, in dem Politik alltäglich ist, d.h. in den Praktiken professioneller Politiker*innen, dem täglichen Politik-Teil der Zeitung oder den eingespielten Routinen von Wahlsonntagen. Vielmehr existiert ein eigenes Register von Praktiken, das sich dadurch auszeichnet, dass es sich verhandelnd - z.B. kritisch, bestätigend, infragestellend - auf andere Praktiken bezieht. Nicht alles ist politisch, aber in diesen Praktiken kann alles politisiert werden.

Um sich diesen Fragen zu nähern, soll ausgehend von der politischen Philosophie Hannah Arendts in Kapitel 2 erläutert werden, in welcher Weise räumliche Bezüge in politischen Praktiken eine zentrale Bedeutung haben und welche Ausgangspunkte sich dabei für eine Geographie politischer Praktiken ergeben. Ausgehend von einer praxistheoretischen Perspektive werden dann in Kapitel 3 politische Praktiken in ihrer Besonderheit charakterisiert. Im Gegensatz zu einigen Vertreter*innen der radikalen Demokratie, die von ,dem Politischen" als einer eigenen ontologischen Kategorie ausgehen (v.a. Badiou und Hallward, 2003; Rancière, 2002; Žižek 1999), kann dann eine nähere Betrachtung politischer Praktiken in Kapitel 4 zeigen, dass sie zwar mit alltäglichen Routinen brechen, zugleich jedoch auf alltäglichen Praxismustern als Medium ihrer Ausdrucksfähigkeit aufbauen.

\section{Eine Geographie politischer Praktiken nach Hannah Arendt}

Kaum eine zweite Denkerin hat sich so intensiv mit dem Wesen politischen Handelns auseinandergesetzt wie Hannah Arendt. Sie schrieb ihr Buch, ,Vita Activa oder vom Tätigen Leben“" (Arendt, 1998b ${ }^{1}$ ) als ein Plädoyer für den Erhalt einer politischen Domäne, in der sich Pluralität, Meinungsverschiedenheit und Streit manifestieren können. In diesem Vorläufer heutiger Praxistheorien unterscheidet Arendt zwischen drei grundsätzlich unterschiedlichen Logiken von Praxis: Arbeiten, Herstellen und Handeln (Arendt, 1998b:16). Arbeiten müssen Menschen aufgrund ihrer biologischen Natur; es dient vor allem der notwendigen Aufrechterhaltung ihres Metabolismus. Dementsprechend werden die Produkte der Arbeit auch immer wieder konsumiert und es entstehen keine bleibenden Produkte (ebd.:99 ff.). Idealtypische Praktiken, die in die Kategorie Arbeit fallen, sind z.B. Putzen und Kochen. Herstellen dagegen schafft bleibende Produkte, die die Welt der Dinge dauerhaft verändern und formen. Während das letzte Ziel von Arbeit das Überleben bzw. die Abwendung des natürlichen Verfalls ist, kann Herstellen als zweckorientiertes Tun sehr unterschiedliche Ziele verfolgen. Entscheidend ist allerdings, dass Herstellen immer Mittel zum Zweck bleibt und niemals aus sich heraus legitime Gründe jenseits seines instrumentellen Charakters geben kann (ebd.:181 ff.).

Handeln, die dritte Form von Praxis, ist die aktive Auseinandersetzung mit Mitmenschen, die die Welt anders sehen und deswegen andere Wünsche und Pläne haben. Nach Arendt beruht unsere grundlegende Fähigkeit zu handeln auf zwei Merkmalen der menschlichen Existenz: Pluralität und Natalität. Pluralität ergibt sich aus der Tatsache, dass "die Menschen, nicht der Mensch, auf der Erde leben und die Welt bewohnen" (ebd.:7). Handeln können und müssen die Menschen auf der Grundlage ihrer Pluralität, innerhalb derer sie immer unterschiedliche Perspektiven auf die gemeinsam geteilte Welt haben. Natalität, d.h. die Tatsache, dass jede Person im Moment ihrer Geburt ein beispielloser und einzigartiger Neuankömmling in der Welt ist, verleiht jeder menschlichen Existenz das Prinzip des Anfangs. Arendt spricht im Hinblick auf das Handeln von einer zweiten Geburt, da es in die gegebene Welt einbricht, indem es diese herausfordert, befragt oder bestätigt.

Dabei besteht der Sinn des politischen Handelns nicht darin, die eigenen Ideen durchzusetzen. Die Idee des souveränen Akteurs hat nichts mit Politik zu tun, da sie der Freiheit widerspricht, wie sie im politischen Handeln ausgeübt wird

\footnotetext{
${ }^{1}$ Obwohl das Buch 1958 zunächst in englischer Sprache unter dem Titel „The Human Condition“ erschienen ist, beziehen sich die meisten Zitate in diesem Artikel auf die deutschsprachige Version aus dem Jahr 1967. Lediglich dort, wo explizit auf Formulierungen der englischsprachigen Originalausgabe (Arendt, 1998a) Bezug genommen wird, wird jene Version zitiert.
} 
(Arendt, 1960). Unter der Bedingung der Pluralität ist Handeln niemals beendet und hat keinen anderen Zweck als das Handeln selbst, das darin besteht, seiner eigenen Position Gehör und Anerkennung zu verschaffen (ebd.:213 ff).

Politik, verstanden als die Sphäre des Handelns, in der bestehende Strukturen thematisiert und kontrovers verhandelt werden, wird nach Arendt zunehmend bedroht durch Logiken, die aus den Bereichen der Arbeit und des Herstellens übernommen wurden (ebd.:278 ff.). Ein Beispiel ist das management-orientierte Verständnis von Politik, in dem ihre vornehmliche Aufgabe in der Herstellung einer besseren Zukunft gesehen wird, deren Leitlinien (z.B. wirtschaftliches Wachstum) allerdings politisch unhinterfragt bleiben. Ein anderes Beispiel ist die Beschwörung einer natürlichen, völkischen Gemeinschaft, die über allen politischen Kontroversen steht und für deren Überleben eine konstante Versorgungsund Reinigungsarbeit notwendig ist. Laut Arendt besteht politisches Handeln eben nicht im Ausführen von Regierungsroutinen und im Befolgen von gegebenen Regeln des politischen Betriebs, sondern in der kreativen, oft kontroversen und dadurch unvorhersehbaren Auseinandersetzung mit der Multiperspektivität, die sich aus der Pluralität der Menschen ergibt. Politisches Sprechen und Handeln (,,speech and action“, Arendt, 1998a:175) bewegt sich im Zwischenraum zwischen den Menschen, verändert das Beziehungsgewebe menschlicher Angelegenheiten und macht damit Personen zu politischen Akteuren.

Welche Möglichkeiten eröffnen sich nun einer Politische Geographie, die Praktiken - anstatt z.B. Macht oder Territorialität - als zentralen Zugangspunkt zu politischen Prozessen wählt? Dieses Thema beinhaltet drei Fragekomplexe: (1) Inwiefern sind Notwendigkeit und Möglichkeit von Politik an die räumlichen Bedingungen menschlicher Existenz gebunden? (2) Inwiefern nehmen politische Praktiken Bezug auf diese Strukturen der Räumlichkeit bzw. welche Räume, Orte und Maßstäbe werden in ihnen konstituiert? (3) Welche Rolle spielt Materialität bei der Stabilisierung, Beschränkung oder Lenkung politischer Praktiken?

\subsection{Politische Praktiken und der geteilte Raum}

Eine Schiffbrüchige, die allein auf einer Insel gestrandet ist, kann nicht handeln: Sie muss arbeiten, um zu überleben, und kann herstellen, um sich eine Welt von Artefakten zu schaffen. Handeln im Sinne Arendts kann sie deshalb nicht, weil Handeln die Konfrontation mit der Alterität anderer Menschen und damit auch deren - zumindest mittelbare - Kopräsenz voraussetzt. Welcher Art diese mittelbare Kopräsenz beschaffen sein muss bzw. in welcher Weise Politik-Machen in virtuellen und physischen Räumen stattfinden kann, soll anhand einer - zugegebenermaßen sehr konstruierten - Variation dieser Schiffbruchsituation verdeutlicht werden: Eine Astronautin ist während ihrer Weltraummission auf einem unbewohnten Planeten gestrandet, der sich in einem räumlich so fernen Bereich des Universums befindet, dass für sie keinerlei Chance mehr besteht, jemals wieder zur Erde oder zu einem anderen von Menschen bewohnten Teil des Universums zurückzukehren. Allerdings verfügt sie über einen hervorragenden, zeitlich kaum verzögerten Internetempfang, da die Übertragung elektromagnetischer Wellen zur Erde sehr gut funktioniert. Inwieweit besteht in dieser Situation für sie die Notwendigkeit bzw. die Möglichkeit politischen Handelns?

Auf dieses Gedankenexperiment sind sehr unterschiedliche Reaktionen möglich: von dem Standpunkt, es gäbe keinen grundsätzlichen Unterschied zwischen dieser spezifischen Situation und der jedes anderen politischen Subjektes, bis zur Einschränkung, die Möglichkeit zu politischem Handeln sei mit der Frage verbunden, inwieweit ihr Computer mit einer funktionierenden Kamera ausgestattet ist, die es der Astronautin erlauben würde, ihre körperliche Existenz filmisch zu repräsentieren. Diese Frage nach den körperlichen und kommunikativen Bedingungen unserer Subjektwerdung findet ihren Ausdruck u.a. in der Debatte um politische Virtualität (vgl. z.B. Barnett, 2004; Castells, 2015; Kitchin, 2016; Elwood und Leszczynski, 2018) und ist bestimmt noch nicht abschließend beantwortet. Im Folgenden sei ein Standpunkt zur Diskussion gestellt, der sich aus den Raumvorstellungen Arendts ergibt.

Der entscheidende Ausgangspunkt ist dabei, dass die Astronautin auf ihrem einsamen Planeten niemanden hat, mit der sie sich ihren Raum teilen muss bzw. kann; die Beziehung zwischen ihr und den Menschen auf der Erde basiert ausschließlich auf Kommunikation, die an keine Ko-Präsenz gebunden ist, und sie kann deshalb ihre Kommunikationspartner*innen nach Belieben an- und abschalten. D.h. sie ist in keiner Weise gezwungen, mit Individuen oder Gruppen zu interagieren, die sie sich nicht selber ausgesucht hat. Ebenso kann jeder Mensch auf der Erde davon absehen, mit ihr zu kommunizieren oder sie überhaupt zur Kenntnis zu nehmen. Dies bedeutet, dass es ihr möglich ist, ganze Teile der Menschheit aus ihrer kommunikativen Welt zu eliminieren und ihre gesamte Kommunikation freiwillig und damit weitgehend irritationsfrei zu gestalten. Konflikten und Auseinandersetzungen mit abweichenden Perspektiven und Weltsichten kann sie vollkommen aus dem Weg gehen.

Kurzum, sie hat keinen ,accidental neighbor" (Massey, 2010:111 ff.), d.h. keine Person mit der sie sich zwangsläufig auseinandersetzen muss, weil sie den physischen Raum mit ihr teilt. Während sich für Massey in dieser Figur vor allem die ,Zufälle des Raumes ' manifestieren, soll an dieser Stelle dagegen das Zwangsläufige und Unerwählte ihrer Präsenz im Vordergrund stehen. Nach Arendt macht uns allein die Tatsache, dass wir uns zwangsläufig auch mit solchen Menschen die Oberfläche der Erde teilen müssen, die wir uns nicht und die uns nicht ausgesucht haben, zu politischen Wesen.

Natürlich gibt es Versuche des räumlichen Ausweichens oder der radikalen Abschottung hinter Mauern (siehe auch z.B. Brown, 2010); letztlich bleibt der physische Raum der Erdoberfläche jedoch immer begrenzt und niemand kann 
dauerhaft in ,splendid isolation' leben. So beginnt Arendt ihr Buch mit einer Kritik an der damaligen Euphorie, die Eroberung des Weltraumes würde der Menschheit die Möglichkeit eines neuen Anfangs im Zusammenleben eröffnen (Arendt, 1998b:7 ff.). Die Suche nach einer neuen Erde sei die falsche Antwort auf die Fragen, die eine „Ethik der Kohabitation“ (Butler, 2012) an uns stellt. Und so leitet auch sie am Ende ihres langen Berichtes über „Eichmann in Jerusalem“ (Arendt, 2011:404) das im Namen der Menschheit gestellte Todesurteil gegen ihn allein aus dem folgenden Umstand ab: Eichmann und seine Mitstreiter hätten geglaubt, sie könnten entscheiden, mit wem sie die Erde teilen wollen und mit wem nicht. Dieses Selbst- und Weltbild markiert für Arendt gewissermaßen den absoluten Nullpunkt aller Politik.

Die Astronautin mit dem Internetzugang muss und kann aus diesem Grund nicht - oder allenfalls eingeschränkt - politisch handeln, denn ihre Existenz liegt jenseits dieser Zwangsjacke begrenzter Räumlichkeit und muss sich dementsprechend auch nicht den Herausforderungen einer Ethik der Kohabitation stellen. Anders ausgedrückt, sie verfügt in ihrer Welt nicht über zu wenig, sondern über zu viel Souveränität. Da in ihrem Handeln ihr Platz in einer mit anderen geteilten Welt nicht wirklich auf dem Spiel steht, ist es ihr nicht möglich, politisch zu handeln.

\subsection{Der Erscheinungsraum als Ort politischer Praktiken}

Die zweite Frage richtet sich an die räumlichen Bezüge, in die politische Praktiken eingelassen sind bzw. die in ihnen hergestellt werden. Bei ihrer Verteidigung des Bereiches der Politik in unserer Gesellschaft greift Arendt immer wieder auf das Vorbild der Polis in der griechischen Antike zurück, wo alle politischen Fragen auf der Agora, dem zentralen öffentlichen Platz, debattiert und entschieden wurden. Dieses Model einer unmittelbaren, auf face-to-face Kontakten aufbauenden und gleichzeitig extrem exkludierenden Demokratie - Frauen und Sklaven blieben ausgeschlossen - kann jedoch in Zeiten von Globalisierung, repräsentativer Demokratie, Massenmedien und Internet wenig Anhaltspunkte dafür geben, wo heute der Raum der Politik ist bzw. sein soll. Wo existiert in unseren Zeiten das konkrete moderne Äquivalent zur antiken Agora? Aus der Perspektive der Praxistheorie lässt sich diese Frage folgendermaßen re-formulieren: Wenn Orte und Räume in und durch Praktiken gebildet werden (Schatzki, 2015), wo finden wir heute jene Praktiken, die damals die Agora bildeten, und welche Orte und Räume bilden diese?

Ein wichtiges Konzept ist für Arendt der sogenannte Erscheinungsraum, d.h. der Raum, ,der dadurch entsteht, dass Menschen voreinander erscheinen, und in dem sie nicht nur vorhanden sind wie andere belebte oder leblose Dinge, sondern ausdrücklich in Erscheinung treten" (Arendt, 1998b:250). Dieser entsteht überall dort, wo Menschen sich in ,speech and action', d.h. in politischen Praktiken, zusammenfinden, und als solcher geht er allen Formen von Staat- lichkeit und politischer Öffentlichkeit voraus. Somit stellt er die Urform bzw. die kleinste Einheit von Öffentlichkeit - verstanden als politischer Raum - dar. Als Raum, der in Praktiken gebildet wird, bleibt er immer an die Aktualisierung dieser Praktiken gebunden und löst sich auf, sobald diese nicht mehr ausgeführt werden. Damit gleicht er jenem Raum, den Menschen, die miteinander reden, zwischen sich bilden; sobald das Gespräch beendet ist, ist auch dieser Raum verschwunden. Dabei ist in jeder Versammlung von Menschen zwar die Möglichkeit, aber nicht die Notwendigkeit eines Erscheinungsraumes gegeben (ebd. 1998b:251).

Politische Räume erscheinen in dieser Perspektive als flüchtige Arrangements, die sich immer und überall dort, und nur dort, auftun, wo und solange im politischen Register gehandelt wird. Tatsächlich gibt es in der Literatur einige Beispiele für die Emergenz von Erscheinungsräumen in Handlungskontexten, die auf den ersten Blick eher unpolitisch wirken. Ein Beispiel ist eine Untersuchung von Arbeitstreffen von Mediziner*innen und AIDS-Aktivist*innen während der 1980er Jahre, in denen aus der anfänglichen Sprachlosigkeit zwischen der explizit emotionslosen Sachlichkeit der Wissenschaft und der existenziellen Dringlichkeit der Betroffenen nach und nach ein politischer Raum entstand, der die offene Konfrontation der verschiedenen Auffassung, aber auch das Erlernen der Sprache und Perspektive der jeweiligen Gegenseite ermöglichte (Topper, 2011). Die Untersuchung solcher Situationen, in denen sich in überraschenden Praktikenzusammenhängen - z.B. im Verwaltungshandeln, in Markttransaktionen oder womöglich sogar in mehrals-menschlichen Kontaktbereichen (Haraway, 2008) zwischen Mensch und Tier - unvermittelt oder allmählich Erscheinungsräume eröffnen, kann eine wichtige Aufgabe einer Geographie politischer Praktiken darstellen.

\subsection{Materialität und politische Praktiken}

Die letzte Frage bezieht sich auf die Rolle von Materialität: In welcher Weise verleihen Körper, Dinge und Orte dem scheinbar so flüchtigen Phänomen der Erscheinungsräume Stabilität bzw. Berechenbarkeit? Welche räumlichen Strukturen legen politische Praktiken nahe bzw. verhindern diese? Aus diesem weiten, vielschichtigen Themenfeld sei an dieser Stelle nur die für Arendt zentrale Unterscheidung zwischen privaten und öffentlichen Räumen herausgegriffen: Während die Öffentlichkeit durch politisches Handeln, Nicht-Exklusivität und Freiheit charakterisiert ist, stellt das Private den Bereich der Notwendigkeit, der Exklusivität und des persönlichen Schutzes dar (Arendt 1998b, S. 62ff.). Gerade von feministischer Seite wurde diese strikte Trennung vielfach scharf kritisiert (z.B. Honig, 1993; Benhabib, 1996), da der private Raum allzu oft als Schutzschild für Praktiken der Gewalt, Ausbeutung und Unterdrückung dient (McKinnon, 1989). Gleichwohl impliziert Arendt keine einfache Dichotomie zwischen Innen und Außen, wenn sie den privaten und den öffentlichen Raum voneinander unterscheidet: Denn 
auch der Bereich der Öffentlichkeit muss sorgfältig eingehegt werden. Arendt verweist in diesem Zusammenhang auf die Mauer, die die griechische Polis umgab, und die damit den öffentlichen Raum, in dem politische Praktiken stattfinden konnten, erst definierte (Arendt, 1998b:73 ff.). Und auch der zentrale Platz der Agora war gewissermaßen ein architektonisch definierter Ort, der der Öffentlichkeit eine Stätte bieten sollte. Diese materielle Einhegung des öffentlichen Raumes hat nach Arendt die Funktion, den politischen Raum und damit die Möglichkeit des Handelns vor dem Übergreifen anderer Logiken der Praxis (Arbeiten, Herstellen) zu schützen.

Auch wenn sich Arendt nur am Rande mit materiellen Raumstrukturen beschäftigt, so liegt es doch auf der Hand, diese beiden Bereiche mit konkreten, materiell verfassten Raumkategorien - z.B. die Straße und das Haus - zu identifizieren. Arendts Aufteilung der Praxis findet sich damit also in einer mehr oder wenigen eindeutigen Zuschreibung von bestimmten offiziellen Stätten des Politik-Machens und damit der Entstehung von Erscheinungsräumen wieder. Der Platz dafür sind beispielsweise Parlaments- oder Regierungsgebäude im Kontext repräsentativer Demokratien oder städtische Plätze und Straßen als Schauplätze politischer Demonstrationen (vgl. z.B. Bar'el, 2017).

Wenn Arendt allerdings zugibt, dass der Bau der Mauer um die Polis für die Griechen ein religiöser und kein politischer Akt war, dann stellt sich die Frage, wie diese Grenze heute, wo sie nicht mehr von außen gestiftet werden kann, gezogen und legitimiert wird. Immerhin stellt diese Frage, welches der Ort der Politik in unserer Gesellschaft sein soll, eine politische Frage par excellence dar! Aus der Sicht eines relationalen bzw. performativen Raumverständnisses erscheinen private und öffentliche Räume als ,normative Regionalisierungen“ (Werlen, 1995:339), die nicht an sich materiell gegeben, sondern immer in Praktiken produziert und re-produziert werden. Der alltäglichen, nicht-reflektiven Praxis bieten sie dabei Stabilität und Selbstverständlichkeit, gerade weil sie mit materiellen Arrangements wie Häusern und Plätzen verbunden werden.

Im politischen Register können jedoch auch diese Räume ihre Selbstverständlichkeit einbüßen und Gegenstand von Interpretation, Infragestellung oder auch expliziter Affirmation werden. Die Grenze von privaten und öffentlichen Räumen ist, wie auch im folgenden Kapitel gezeigt werden soll, keinesfalls in Stein gemeißelt, sondern immer offen für kleine und große Infragestellungen und Reformulierungen. Die Aneignung von und der Rückzug aus alltäglichen Räumen, das Ziehen von Grenzen und deren Überschreitung, die Bestätigung oder Infragestellung territorialer Zuschreibungen: Politische Praktiken sind nicht nur das Vehikel für die Neuvermessung räumlicher Zuschreibungen, sondern bilden ihrerseits neue Räume, die neue Subjektpositionen ermöglichen bzw. provozieren. Eine Geographie politischer Praktiken kann anhand von konkreten Beispielen sehr genau und differenziert analysieren, in welchen Kontexten und durch welche Praktiken Erscheinungsräume entstehen, in welcher Weise alltägliche Raumzuschreibungen in ihnen wirksam sind bzw. von ihnen herausgefordert werden und welche Bedeutung dabei der materiellen Welt der Körper und Dingen zukommt.

\section{Politische Praktiken: Reflexiv, perspektivisch, expressiv}

Obwohl Arendts politische Theorie die Praxis in den Mittelpunkt des Interesses rückt, bleiben ihre analytischen Kategorien menschlichen Tuns - Arbeiten, Herstellen und Handeln - relativ undifferenziert. In dieser Hinsicht bieten aktuelle Praxistheorien (vgl. als Überblick z.B. Hillebrand, 2014; Schäfer, 2014; Everts und Schäfer, 2019) ein wesentlich differenzierteres Instrumentarium zur Untersuchung von Praktiken. Viele praxistheoretische Ansätze (z.B. Giddens, 1984; Bourdieu, 1976; Schatzki, 2002) haben allerdings in der Vergangenheit vor allem die strukturierende Funktion von Praktiken betont und dabei ihr kritisches und widerständiges Potenzial übersehen (Alkemeyer et al., 2017). In seiner Soziologie der Kritik unternimmt Luc Boltanski (Boltanski, 2013) den Versuch, diesem Bild der unentrinnbaren Herrschaft der Routinen eine Analyse tatsächlicher Situationen von Kritik entgegenzusetzen. Dabei unterscheidet er zwischen einem pragmatischen und einem metapragmatischen Register von Praktiken. Im pragmatischen Register ist die praktische Aufmerksamkeit auf das, ,was zu tun ist' gerichtet. Das Tun wird vorwiegend geleitet von Gewohnheiten, d.h. Regelmäßigkeiten, die nicht direkt auf normative Regeln zurückgehen. Dispute gilt es zu vermeiden, weswegen die Toleranz gegenüber Differenzen oder internen Widersprüchen, die sich innerhalb von Praktiken ergeben, hoch ist. Im pragmatischen Modus werden Pluralität und die Unvereinbarkeit verschiedener Wahrheitsansprüche nicht thematisiert und Sprache fungiert als ein unproblematisches System von direkten Entsprechungen zwischen Begriffen und der Realität (ebd.:62 ff.). Dagegen ist im metapragmatischen Register diese stabile Funktionsweise von Sprache nicht mehr automatisch gegeben, und die praktische Aufmerksamkeit bezieht sich weniger auf das Erledigen von Aufgaben, sondern auf die Angemessenheit bzw. Legitimität von Situationsbeschreibungen und Problemdefinitionen (,Ist das wirklich ein Fall von X?'). Routinisierung, wie sie gerade das pragmatische Register kennzeichnet, steht im metapragmatischen Modus zur Disposition, d.h. sie wird kritisiert, infrage gestellt oder aber legitimiert (ebd.:67 ff.).

Wodurch zeichnen sich nun Praktiken aus, die in diesem metapragmatischen Register stehen? Welches Tun kann als speech and action gelten? Von der Beantwortung dieser Frage hängt es ab, ob und in welcher Weise sich Praxistheorien erweitern und als Ausgangspunkt für eine Geographie politischer Praktiken verwenden lassen. Zwei Punkte können bei diesen Überlegungen als Ausgangspunkte fungieren: 
Zum einen gibt es keine den Handlungselementen unmittelbar innewohnenden Charakteristika, die politische Praktiken eindeutig als solche auszeichnen würden. Ein und derselbe Akt kann in einer Situation eindeutig Teil einer politischen Praktik sein und in einer anderen nicht. Zum anderen spielen die Kriterien von Scheitern und Erfolg einer Praktik, d.h. das was Schatzki (2002) ihre teleoaffektive Struktur nennt, eine zentrale Rolle. Es kommt nicht nur darauf an, was jemand tut, sondern ebenso warum!

Anscombes klassisches handlungstheoretisches Konzept intentionaler Handlungen kann dies näher beleuchten (Anscombe, 1957): Ein und dasselbe Tun kann sehr unterschiedlich beschrieben werden (z.B. „Er krümmt den Zeigefinger“, „Er gibt einen Schuss mit einer Pistole ab“, ,Er erschießt Kronprinz Ferdinand“, „Er löst den Ersten Weltkrieg aus“). Es gibt allerdings nur eine Beschreibung (,Er erschießt Kronprinz Ferdinand“), die alle anderen Beschreibungen in sich aufnimmt; jene sind dann lediglich mögliche Antworten auf Fragen nach dem Wie des Handlungsvollzugs bzw. nach den nicht-intendierten Folgen der Tuns. Diese eine Beschreibung ist nicht austauschbar, denn sie benennt die eigentliche Absicht des Tuns (ebd.:37 ff.), d.h. die zutreffende Antwort auf die Frage ,Was tust du?“. Damit impliziert Anscombe zum einen, dass Intentionen keine Privatangelegenheit des handelnden Individuums darstellen, sondern immer eingebettet sind in einen sprachlich und sozial strukturierten, räumlich und zeitlich lokalisierten Bereich der Beschreibbarkeit (Hu, 2017:102), eine Idee, die sich auch mit der praktikentheoretischen Auffassung vereinbaren lässt, nach welcher Praktiken mit ihren teleoaffektiven Strukturen keine psychologischen, sondern soziale Phänomene darstellen. Zum anderen wird aber auch deutlich, dass unterschiedliche Beschreibungen einer Handlung mehr oder weniger zutreffen bzw. den Kern einer Handlung besser oder schlechter treffen können. Die Frage ist nun, in welcher Hinsicht sich politische Praktiken hinsichtlich ihrer solcherart treffenden Beschreibungen untereinander gleichen.

An dieser Stelle seien drei einfache Charakteristika vorgeschlagen, die diese Voraussetzung erfüllen sollen. Diese drei Dimensionen sind dabei als sowohl elementar als auch vollumfänglich gemeint, d.h. alle weiteren möglichen Kriterien (z.B. Machtrelevanz, Staatsbezug, Territorialität) sollen aus ihnen abgeleitet werden können. Wenn diese drei Kriterien auch auf Praktiken zutreffen, die normalerweise nicht zum Spektrum politischen Handelns gezählt werden (z.B. bestimmte künstlerische oder wissenschaftliche Praktiken), dann widerspricht dies nicht dieser Einordnung, sondern zeigt vielmehr, dass Politik - verstanden als metapragmatische Praxis - an vielen Orten und in vielen sozialen Kontexten stattfindet, deren politischer Gehalt in der Regel übersehen wird:

1. Reflexivität: Es sind Praktiken, die sich intentional auf die Beeinflussung anderer Praktiken beziehen. Wie alle anderen Praktiken sind sie einerseits Teil des bestehen- den Gewebes von Praktiken, das die soziale Welt darstellt (vgl. Schatzki, 2002, 2010); andererseits zielen sie zugleich in ihrer teleoaffektiven Struktur auf die Veränderung bzw. Stabilisierung der Topologie ebendieses Gewebes ab. Eine solche Reflexivität ist allerdings nicht nur ein Charakteristikum politischer Praktiken; Praktiken des Lehrens oder der Planung (vgl. Dünckmann et al., 2019) stellen z.B. ebenfalls solche ,Praktiken zweiten Grades ‘ dar.

2. Perspektivität: Politische Praktiken beziehen sich auf die Multiperspektivität und Pluralität der sozialen Welt und damit auf die Möglichkeit des Unvernehmens zwischen Menschen (vgl. Rancière, 2002). Indem sie z.B. Forderungen nach Anerkennung, Gerechtigkeit oder Partizipation ausdrücken, zielen sie auf den Zwischenraum menschlicher Beziehungen ab, der nach Arendt die Menschen gleichzeitig verbindet und trennt.

3. Expressivität: Sie sind verortet und verorten sich in einer Öffentlichkeit bzw. beziehen sich auf diese. Sie zielen nicht nur darauf ab, etwas Bestimmtes zu tun, sondern ebenso, dieses Tun sicht- und hörbar zu machen. In diesem Sinne unterscheiden sie sich von Praktiken wie z.B. reiner Sabotage oder der Verbreitung von Terror, die womöglich einem machtstrategischen Motiv (z.B. Kampf um territoriale Kontrolle) folgen, die aber dennoch nicht darauf angelegt sind, Kritik oder Bestätigung der sozialen Welt zum Ausdruck zu bringen (vgl. bspw. Mustafa et al. (2013) zum Terrorismus).

Zwei kurze Schlaglichter auf konkrete Momente des Politischen sollen diese drei Dimensionen illustrieren: Die erste Situation ist in einem Foto aus Teheran festgehalten, welches zum Weltpressefoto des Jahres 2010 gekürt wurde ${ }^{2}$. Es wurde während der Grünen Revolution aufgenommen, bei der damals in Teheran tausende Menschen gegen die Wiederwahl von Präsident Ahmadinejad protestierten. Allerdings zeigt das Bild keine zornigen Demonstrant*innen, sondern ein idyllisch anmutendes, intimes Bild von Wohnhäusern im warmen Abendlicht. Man sieht die Lichter in den Fenstern der Wohnhäuser und drei Frauen, die auf dem Dach eines dieser Häuser stehen. Eine von ihnen hat die Hände um den Mund gelegt, um etwas in den Abend hinaus zu rufen. In diesen Tagen hatte die brutale Niederschlagung der Massendemonstrationen begonnen und die Menschen suchten sich andere Formen, um ihrer Wut und ihrem Protest Ausdruck zu geben. Täglich, wenn die Stadt langsam zur Ruhe kam, störten sie die abendliche Ruhe, indem sie ,Allahu Akbar (Gott ist groß) aus den Fenstern und von den Dächern ihrer Wohnhäuser riefen.

\footnotetext{
${ }^{2}$ Dieses Foto von Pietro Masturzo ist z.B. auf der Website der World Press Photo Foundation $\mathrm{zu}$ sehen: https://www.worldpressphoto.org/collection/photo/2010/30434/1/ 2010-Pietro-Masturzo-WY (letzter Zugriff: 14 Mai 2020).
} 
In diesem Tun treten die drei Charakteristika politischer Praktiken offen zu Tage. Es ist reflexiv, denn es bezieht sich auf einen Teil des Gewebes sozialer Praktiken, Herrschaftspraktiken im Iran, und will diese verändern. Es ist perspektivisch, denn es bewegt sich in einem Feld, das von der Unvereinbarkeit verschiedener Perspektiven, Demokratie vs. autoritäre Herrschaft, geprägt ist. Es ist expressiv, indem es darum geht, der eigenen Position hör- bzw. sichtbar Ausdruck zu verleihen.

Dass politische Praktiken allerdings auch sehr viel subtiler und weniger offensichtlich sein können, zeigen im zweiten Beispiel Häkli und Kallio (2014) anhand einer kurzen Szene aus dem Film „Fanny und Alexander“ des schwedischen Regisseurs Ingmar Bergman: Alexander wurde von seinem strengen Stiefvater, einem Pastor, für sein ungehöriges Verhalten bestraft. In dem anschließenden Vier-Augen-Gespräch fragt der Stiefvater, ob Alexander denn auch wisse, warum er ihn eben bestraft habe. Alexander antwortet darauf: „Ich glaube, dass Sie Alexander nicht besonders mögen“. So unspektakulär diese Antwort auch ist: Häkli und Kallio sehen in dieser minimalen Verschiebung der normalen Sprachpraxis, indem Alexander von sich selber in der dritten Person spricht, den ganzen politischen Gehalt dieses Momentes. Die ursprüngliche Praktik des väterlichen Ins-Gewissen-Redens, die der Stiefvater ausführt, stellt diese beiden Personen, Vater und Sohn, ins Zentrum eines Arrangements, in dem eigentlich die Intimität des häuslichen Rahmens - die Abwesenheit fremder Personen, die vier Wände des Zimmers, die geschlossene Tür - eine entscheidende Rolle spielt. Diese Szene stellt gewissermaßen ein Idealbild einer privaten Situation dar. Alexander durchbricht diskursiv diese von väterlicher Herrschaft und Intimität geprägte Praktik, indem er verbal die Position einer dritten Person einnimmt. Durch diese kleine Variation der Sprache bezieht Alexander nun eine, körperlich zwar nicht anwesende, aber dennoch rhetorisch wirkungsvolle, Außenperspektive in das Arrangement ein und beschwört damit eine virtuelle Öffentlichkeit herauf. Seine diskursive Praktik ist reflexiv, denn er bezieht sich kritisch auf die häuslichen Praktiken, die ihn umgeben; sie ist perspektivisch, denn sie bewegt sich im bzw. zielt auf den Bereich des Unvernehmens zwischen ihm und seinem Stiefvater; sie ist expressiv, denn sie verleiht - wenn auch indirekt seiner Position Ausdruck. Mit diesem unscheinbaren Satz an diesem unspektakulären Ort praktiziert Alexander eindeutig jene Form von ,speech and action', die Arendt als politisches Handeln auffasst.

Gleichzeitig zeigen diese beiden Beispiele, dass die grobe Unterscheidung zwischen Privatheit und Öffentlichkeit nicht einfach mit dem Haushalt und der Straße bzw. dem Platz gleichgesetzt werden kann, wie dies Arendt nahezulegen scheint. Obwohl beide Situationen sich innerhalb der Grenzen des Haushaltes - innerhalb der ,eigenen vier Wän$\mathrm{de}^{\text {' }}$ - abspielen, entsteht im Vollzug der politischen Praktiken, die dort stattfinden, eine Form von Öffentlichkeit, die im Arendt'schen Sinne als Erscheinungsraum bezeichnet wer- den kann. Die Geographie öffentlicher und privater Räume ist also nicht in Stein gemeißelt. Sie wird in und durch Praktiken im pragmatischen Modus stabilisiert und kann dementsprechend jederzeit durch politische Praktiken im metapragmatischen Modus herausgefordert und aufgebrochen werden.

Mit den drei Dimensionen Reflexivität, Perspektivität und Expressivität existieren Kriterien, mit deren Hilfe politische Praktiken als solche identifiziert, analysiert und letztendlich als Ausgangspunkt einer Geographie politischer Praktiken genommen werden können. Es bleibt allerdings die Frage, in welcher Beziehung politische Praktiken zu all den anderen, nicht-politischen Praktiken stehen und in welcher Weise sich soziale Interaktionen bzw. gesellschaftliche Strukturen im Spannungsfeld zwischen diesen beiden (pragmatisch - metapragmatisch bei Boltanski) bzw. drei (Arbeit - Herstellen - Handeln bei Arendt) Modi menschlicher Praxis herausbilden und wandeln.

\section{Politische Praktiken zwischen Abgrund und Alltag}

Wenn die soziale Welt in ihrer Gesamtheit ein Gewebe von Praktiken darstellt, die auf vielfältige Art und Weise aufeinander bezogen sind (Schatzki, 2010, 2019), dann sind politische Praktiken auf der einen Seite selbst Teil dieses Gewebes und stellen damit ihrerseits den Kontext für andere Praktiken dar. Auf der anderen Seite heben sie sich aber in ihrer Reflexivität, Perspektivität und Expressivität von den gewöhnlichen Praktiken des Alltags ab. In welcher Beziehung stehen dann diese beiden Register von Praxis zueinander? Inwieweit sind Arendts drei Formen der Praxis - Arbeit, Herstellen und Handeln - tatsächlich in verschiedenen Bereichen des sozialen Lebens lokalisiert? Gibt es einen grundlegenden Unterschied zwischen einer ,gesellschaftlichen“ Domäne, in der soziale Institutionen unhinterfragt wirksam sind, und dem Bereich des ,Politischen', dem Bereich menschlicher Interaktion, in dem nicht Regeln und Routinen, sondern Kritik, Streit und Entscheidungen die soziale Praxis bestimmen? Und wenn dies der Fall ist, in welcher Beziehung - gegensätzlich oder komplementär - stehen beide zueinander?

Gesellschaften sind immer wieder mit konfliktgeladenen Situationen konfrontiert, in denen es keine inhaltlichen, verfahrenstechnischen oder diskursiven Regeln mehr gibt, die zur Beilegung des Streites herangezogen werden könnten, weil ebendiese Regeln selbst Gegenstand der Auseinandersetzung sind. Solche Krisen machen deutlich, dass allen politischen Systemen ein unauflöslicher Widerspruch zwischen der Macht der Regeln und der Macht, neue Regeln aufzustellen, d.h. zwischen der konstituierenden und der konstituierten Macht, zu Grunde liegt. Dieses Paradox wird z.B. sichtbar, wenn Demokratien mit antidemokratischen Bestrebungen konfrontiert werden. Sollen diese als Teil des Streits von Ideen gelten, der Demokratien als solche auszeichnet? Oder sollen sie von der politischen Willensbildung ausgeschlos- 
sen werden, auch wenn die Gefahr besteht, damit genau jene demokratischen Prinzipien zu verletzen, die eigentlich verteidigt werden sollen? In diesen Momenten der politischen Konfrontation zeigt sich, dass Gesellschaften nicht auf einem soliden Fundament aus metaphysisch abgesicherten Gründen aufbauen, sondern dass sie den Abgrund der ihr zugrundeliegenden Grundlosigkeit immer nur mehr oder weniger verdecken können (Marchart, 2011). Politische Praxis ist demnach Handeln ohne Letztbegründung: Am Anfang steht allein die Praxis; die abstrakten Prinzipien, die diese Praxis eigentlich vorgängig leiten sollten, werden in ihrem Vollzug erst konstruiert. Michael Oakeshott findet dafür das eingängige Bild des ufer- und grundlosen Ozeans: "In political activity, then, men (sic!) sail a boundless and bottomless sea; there is neither harbor for shelter nor floor for anchorage, neither starting place nor a pointed destination... And if it suggests that politics are nur für die Schwindelfreie (sic!), that should depress only those who have lost their nerve" (Oakeshott, 1991).

Deliberative Theorien (z.B. Benhabib, 2017; Habermas, 1995; Rawls, 1971) unterstreichen in diesem Kontext die Bedeutung von Fairness, Vernunft und Kommunikation, die es ermöglichen sollen, auch angesichts solcher Begründungskrisen einen Konsens zu finden. Dagegen vertrauen Vertreter*innen der radikalen Demokratie (z.B. Rancière, 2002; Badiou, 2003; Žižek, 1999) nicht auf diese Möglichkeit zur Einigung und streben sie auch nicht an, sondern sehen gerade in diesen Momenten des grundsätzlichen und unauflöslichen Unvernehmens den eigentlichen Kern einer ontologischen Kategorie ,des Politischen'.

Agonistische Demokratietheorien (z.B. Honig, 2009; Mouffe, 2013; Tully, 2008 als Überblick Wenman, 2013) bieten dagegen einen Weg an, wie dieser Widerspruch zwischen konstituierender und konstituierter Macht in eine praxistheoretische Sicht auf die Gesellschaft integriert werden kann. Gemeinsam mit den Vertreter*innen der radikalen Demokratie erkennen sie auf der einen Seite die Unmöglichkeit einer Letztbegründung von Regeln an und sehen Dissens und Konflikt als die treibenden Kräfte von Politik. Auf der anderen Seite wollen sie aber ,echte " politische Momente nicht auf die wenigen revolutionären Situationen beschränken, in denen die Gesamtheit aller Regeln zu Disposition gestellt wird. Vielmehr heben sie hervor, dass in politischen Momenten niemals alle bestehenden Regeln zur gleichen Zeit, sondern immer nur bestimmte Ausschnitte der sozialen Welt thematisiert werden. Selbst revolutionäre Bewegungen brechen nicht mit allen gesellschaftlichen Strukturen gleichzeitig, sondern berufen sich immer auf ein Set von gemeinsam geteilten Werten, die es gilt, gegen die bestehenden Herrschaftsverhältnisse endlich zu etablieren oder wieder zu restaurieren. Und indem sich politische Praktiken auf nur einen Bereich der sozialen Welt ausrichten und ihn in das Zentrum der Aufmerksamkeit stellen, wird gleichzeitig die Gültigkeit des restlichen Teils der Gesellschaft, der nicht thematisiert wird, für den Moment stabilisiert. Allein aufgrund der Tatsache, dass unsere kognitive Aufmerksamkeit immer gerichtet ist und wir, indem wir uns einer Sache zuwenden, alles andere in diesem Augenblick gewissermaßen gedanklich fallen lassen, ist jede politische Thematisierung immer mit einer De-Thematisierung und Normalisierung aller anderen Bereiche des sozialen Lebens verbunden (vgl. Hannah, 2019).

Politische Praktiken können dann als eine Variation bzw. eine Verschiebung alltäglicher Praktiken aufgefasst werden. Dabei ist die Verstehbarkeit von Praktiken ein zentraler Punkt. Politische Praktiken sind nicht dann als gescheitert anzusehen, wenn sie ihr Endziel nicht erreichen und die in ihnen intendierte Änderung der sozialen Welt nicht stattfindet. Vielmehr liegt nach Arendt der Sinn politischer Praxis vor allem in ihrem Vollzug, d.h. darin, die eigene Stimme zu erheben bzw. einen bestimmten Standpunkt zu vertreten. Wenn allerdings dieses Handeln überhaupt nicht - d.h. auch nicht von einem möglichen Opponenten - als politische Praxis in ihrer Reflexivität, Perspektivität und Expressivität wahrgenommen wird, dann muss die Praktik in der Tat als gescheitert gelten: Wenn die Nachbarn der protestierenden Frauen in Teheran sich über deren Frömmigkeit freuen; wenn der Stiefvater von Alexander denkt, dass dieser nicht einmal grammatikalisch korrekte Sätze bilden kann; wenn also der jeweilige Protest gar nicht als solcher wahrgenommen wird, ist er gescheitert. Gelungene politische Praktiken müssen als, speech and action' im Sinne Arendts erkennbar sein. Und wenn Verstehen und Missverstehen in und durch Praktiken stattfindet, müssen sich auch politische Praktiken immer im Bereich öffentlicher Verstehbarkeit bewegen.

Praktikentheoretisch ausgedrückt bedeutet dies, dass das Gewebe von Praktiken, das die soziale Welt darstellt, durchsetzt ist mit solchen, die sich in ihrem Vollzug auf die Topologie dieses Gewebes beziehen und dabei gewollt oder ungewollt seine Grundlosigkeit offenbaren, ohne dabei jemals vollständig mit ihm brechen zu können. Die Öffnungen, in denen eine solche Kritik (oder Bekräftigung) möglich ist, ergeben sich nicht trotz der kollektiven Bindungskraft von Praktiken, sondern gerade wegen ihnen. Gerade die Bruchlinien zwischen unterschiedlichen Praktiken und sozialen Ordnungen, die jeweils ihre eigene Logik haben, sind jene Orte, an denen inmitten des Gewebes sozialer Praktiken Subjekte entstehen können, denen es möglich ist, Kritik zu üben bzw. in einem metapragmatischen Modus zu handeln (Alkemeyer et al., 2017).

Darüber hinaus wird Kritik nur durch kollektiv geteilte Praktiken verständlich und damit erst möglich. Dies betrifft nicht allein solche politischen Praktiken, die für uns ohne weiteres als solche erkennbar sind, wie z.B. Reden halten, demonstrieren oder Petitionen unterschreiben. Ebenso gilt dies für solche, deren politische Ausrichtung nicht unmittelbar erkennbar ist und die eher subtil verstehbar sind. Dass beispielsweise bolivianische Bauern ihren Marktstand mit großen Fotos schmücken, die sie bei der landwirtschaftlichen Arbeit zeigen (vgl. Dünckmann und Fladvad, 2016), hat auf den ersten Blick wenig politischen Gehalt. Allerdings dienen diese Fotos weniger als Werbemaßnahme zur Absatzsteige- 
rung, sondern verleihen vielmehr der Forderung der Bauern Ausdruck, dass ihre Arbeit auch über den strikten Marktwert ihrer Produkte hinaus wertgeschätzt wird. Auf diese Art können auch scheinbar alltägliche, allgemein verständliche Praktiken (z.B. Fotografieren, Einen-Marktstand-Betreiben) eine grundsätzliche Kritik an der Funktionsweise kapitalistischer Märkte und an dem Fetischcharakter ihrer Waren transportieren. Durch eine praxistheoretische Sichtweise ließe sich zeigen, an welchen überraschenden Orten diese erscheinen können und auf welche vielfältige Art und Weise sie mit den gewöhnlichen Praktiken des Alltags verbunden sind.

\section{Fazit}

Eine Geographie politischer Praktiken, wie sie sich aus einer Verbindung der Ideen Hannah Arendts mit Ansätzen der Praxistheorie entwickeln lässt, eröffnet die Möglichkeit, traditionelle Themen der Politischen Geographie neu zu fassen und neue Perspektiven auf scheinbar bekannte Phänomene zu entwickeln. Dies zeigen geographische Arbeiten aus den letzten Jahren, die, obwohl sie das Label nicht benutzen und sich nicht zwangsläufig auf Arendt beziehen, kompatibel mit den hier vorgestellten Grundideen sind: Petermann (2007) untersucht z.B. die ritualisierten Gedenkzeremonien an den Ersten Weltkrieg als eine politisch besonders relevante Kategorie von politischen Praktiken, die der Affirmation bestehender Machtstrukturen dienen. Sowohl Weisser (2014; siehe auch Weisser und Müller-Mahn, 2017) als auch Schäfer (2015) richten einen mikro-geographischen Blick auf Praktiken der Aushandlung, Formulierung und Übersetzung von internationalen und nationalen Politikstrategien im $\mathrm{Zu}$ sammenhang mit dem Klimawandel. Fladvad (2017) nimmt Ernährungssouveränität als eine neue politische Rechtsforderung unter die Lupe und analysiert die Manifestierung der damit verbundenen Gerechtigkeitsvorstellungen in alltäglichen landwirtschaftlichen Praktiken. Schurr (2013) nähert sich dem traditionellen Feld der Wahlgeographie auf eine neue Weise, indem sie die konkrete körperliche und emotionale Praxis von Wähler*innen, Demonstrant*innen und Politiker*innen genau untersucht und die Entstehung neuer Mikro-Räume des Politischen identifiziert. Dies sind nur einige Beispiele für die Bandbreite und Vielfalt der Phänomene, die sich mit Hilfe einer Geographie politischer Praktiken analysieren lassen.

Dieser Ansatz kann politische Praktiken in ihrem gesamten Spektrum des metapragmatischen Registers in den Blick nehmen, d.h. sich nicht nur mit dem Protest, sondern auch mit der Affirmation von herrschenden Machtstrukturen beschäftigen. In welchen Praktiken manifestiert sich die aktive Bestätigung, Legitimierung oder gar Überhöhung bestehender Machtstrukturen? In welche Raumbezüge und materiellen Arrangements sind diese eingebunden? Und welche Bedeutung haben materielle Arrangements bei der DePolitisierung und praktischen Stabilisierung von ehemals po- litisch diskutierten Themen, d.h. beim Übergang vom metapragmatischen zum pragmatischen Modus von Praxis?

Ein weiterer Untersuchungsbereich könnte der zunehmende politische Gehalt von bestimmten Praktiken sein (vgl. auch Everts et al., 2019), wie das Beispiel des Fahrradfahrens zeigt. Durch Konzepte wie bicyle citizenship oder critical mass hat hier eine Praktik eine eigene politische Stimme in der politischen Arena der Stadtentwicklung entwickelt, die mit einer starken Identitätsbildung und Distinktion verbunden ist. Die Forderungen von Fahrradfahrer*innen gegenüber der Planung tragen Züge einer politischen Auseinandersetzung, bei der es gleichermaßen um Nachhaltige Mobilität wie um Identitätspolitik und soziale Gerechtigkeit geht.

Eine harmoniesüchtige Gesellschaft, die soziale Kohärenz als absolutes Ziel ansieht und inhaltliche Konflikte und Krisen scheut, ist nach Ansicht Boltanskis (2013:155) pathologisch. Vielmehr sollte die soziale Realität in ihrer Fragilität und Unfertigkeit erkannt und geschätzt werden (ebd.:218). Eine Geographie politischer Praktiken lenkt den Blick auf die vielfältigen und oftmals überraschenden Erscheinungsräume, die sich dort auftun, wo diese Ungewissheit und metaphysische Bodenlosigkeit unserer sozialen Welt zutage tritt und verhandelt wird. Dies sind die Momente, in denen die soziale Welt aktiv und bewusst verändert wird. $\mathrm{Zu}$ zeigen, dass dies nicht nur ,für Schwindelfreie ' gilt und keinen hehren Heroismus verlangt, sondern in der Regel eingebunden ist in unser alltägliches Tun und an alltäglichen Orten stattfindet, kann ein wichtiger Beitrag einer Geographie politischer Praktiken sein.

Datenverfüg barkeit. Für diesen Artikel wurden keine Datensätze genutzt.

Interessenkonflikt. Der Autor erklärt, dass kein Interessenkonflikt besteht.

Danksagung. Ich bedanke mich bei den beiden anonymen Gutachter*innen sowie bei Jonathan Everts, Simon Runkel, Sebastian Ehret und Jens Reda für ihre kritisch-konstruktiven Kommentare.

Begutachtung. This paper was edited by Benedikt Korf and reviewed by two anonymous referees. 


\section{Literatur}

Alkemeyer, T., Buschman, N., and Michaeler, M.: Critique in praxis: Arguments for a subjectivation theoretical expansion on practice theory, in: Praxeological political analysis, Routledge advances in sociology, 196, edited by: Michael, J. and Littig, B., Routledge, London, New York, 67-84, 2017.

Anscombe, G. E. M.: Intention, Harvard University Press, Cambridge, Massachusetts, London, 1957.

Arendt, H.: Freedom and Politics: A lecture, Chicago Review, 14, 28-46, 1960.

Arendt, H.: The human condition, 2nd Edn., University of Chicago Press, Chicago, 1998a.

Arendt, H.: Vita activa oder Vom tätigen Leben, 10th Edn., Piper, München, 1998b.

Arendt, H.: Eichmann in Jerusalem, Ein Bericht von der Banalität des Bösen, Piper, München, Zürich, 2011.

Badiou, A. and Hallward, P.: Über Metapolitik (TransPositionen, 12), diaphanes, Zürich, 2003.

Bar'el, Z:: Tahrir Square, From place to space, The geography of representation, Middle East J., 71, 9-22, https://doi.org/10.3751/71.1.11, 2017.

Barnett, C.: Media, democracy and representation: Disembodying the public, in: Spaces of democracy. Geographical perspectives on citizenship, participation and representation, edited by: Barnett, C. and Low, M., Sage, Thousand Oaks, London, 185-206, 2004.

Barnett, C.: Situating the geographies of injustice in democratic theory, Geoforum, 43, 677-686, https://doi.org/10.1016/j.geoforum.2011.03.002, 2012.

Benhabib, S.: The reluctant modernism of Hannah Arendt, Sage, Thousand Oaks, 1996.

Benhabib, S.: Kritik, Norm und Utopie. Die normativen Grundlagen der Kritischen Theorie, Fischer, Frankfurt am Main, 2017.

Boltanski, L.: On critique. A sociology of emancipation, Wiley, Hoboken, 2013.

Bourdieu, P.: Entwurf einer Theorie der Praxis auf der ethnologischen Grundlage der kabylischen Gesellschaft, Suhrkamp, Frankfurt am Main, 1976.

Brown, W.: Walled states, waning sovereignty, Zone Books, MIT Press, New York, Cambridge, Massachussets, 2010.

Butler, J.: Precarious life, vulnerability, and the ethics of cohabitation, J. Speculat. Philos., 26, 134-151, 2012.

Cassegård, C.: Contestation and bracketing: the relation between public space and the public sphere, Environ. Plan D, 32, 689703, https://doi.org/10.1068/d13011p, 2014.

Castells, M.: Networks of outrage and hope. Social movements in the Internet Age, 2nd Edn., Polity Cambridge, UK, Malden, MA, USA, 2015.

Collins, M.: Conflict and contact: the ,humane' city, agonistic politics, and the phenomenological body, Environ. Plan. D, 28, 913 930, https://doi.org/10.1068/d13209, 2010.

Conolly, W. E.: A world of becoming, Duke University Press, Durham, NC, 2011.

Debarbieux, B.: The political meaning of landscape (through the lens of Hannah Arendt's The human condition), in: The place of landscape: Concepts, contexts, studies, edited by: Malpas, J., MIT Press, Cambridge, MA, 131-149, 2011.
Dikeç, M.: Politics is sublime, Environ. Plan. D, 30, 262-279, https://doi.org/10.1068/d12610, 2012a.

Dikeç, M.: Space as a mode of political thinking, Geoforum, 43, 669-676, https://doi.org/10.1016/j.geoforum.2012.01.008, 2012b.

Dikeç, M.: Beginners and equals: Political subjectivity in Arendt and Rancière, Trans. Inst. Brit. Geogr., 38, 78-90, https://doi.org/10.1111/j.1475-5661.2012.00508.x, 2013.

Dünckmann, F. and Fladvad, B.: The practice of changing the rules of practice: An agonistic view on food sovereignty, Geogr. Z., 104, 25-49, 2016.

Dünckmann, F., Haubrich, D., and Winkler, J.: Praktiken und Planung, in: Handbuch Praktiken und Raum: Humangeographie nach dem Practical Turn, Herausgeber: Everts, J. und Schäfer, S., Transcript, Bielefeld, 317-340, 2019.

Elwood, S. and Leszczynski, A.: Feminist digital geographies, Gender Place Cult., 25, 629-644, https://doi.org/10.1080/0966369X.2018.1465396, 2018.

Everts, J. and Schäfer, S. (Hrsg.): Handbuch Praktiken und Raum: Humangeographie nach dem Practical Turn, Transcript, Bielefeld, 2019.

Everts, J., Geiselhart, K., Rominger, S., and Winkler, J.: Praktiken des Regierens, dominante Projekte, Verantwortung: praxeologische Zugänge zum Thema Macht, in: Handbuch Praktiken und Raum: Humangeographie nach dem Practical Turn, Herausgeber: Everts, J. und Schäfer, S., Transcript, Bielefeld, 223-244, 2019.

Fladvad, B.: Topologien der Gerechtigkeit. Eine politischgeographische Perspektive auf das Recht auf Ernährungssouveränität in Boliven, Kieler Geographische Schriften 130, Geographisches Institut Kiel, Kiel, 2017.

Fraser, N.: Unruly practices: Power, discourse, and gender in contemporary social theory, University of Minnesota Press, Minneapolis, 1989.

Giddens, A.: The constitution of society. Outline of the theory of structuration, Polity Press, Cambridge, 1984.

Habermas, J.: Theorie des kommunikativen Handelns, Suhrkamp, Frankfurt am Main, 1995.

Häkli, J. and Kallio, K. P.: Subject, action and polis. Theorizing political agency, Prog. Hum. Geogr., 38, 181-200, https://doi.org/10.1177/0309132512473869, 2014.

Hannah, M. G.: Direction and socio-spatial theory: A political economy of oriented practice, Routledge, London, New York, 2019.

Haraway, D. J.: When species meet, University of Minnesota Press, Minneapolis, 2008.

Hillebrandt, F.: Soziologische Praxistheorien. Eine Einführung, Springer VS, Wiesbaden, 2014

Honig, B. (Ed.): Feminist interpretations of Hannah Arendt, Pennsylvania State University Press, University Park, PA, 1993.

Honig, B.: Emergency politics. Paradox, law, democracy, Princeton University Press, Princeton, 2009.

$\mathrm{Hu}$, C.: A jungle that is continually encroaching. The time of disaster management, Environ. Plan. D, 36, 96-113, https://doi.org/10.1177/0263775817729377, 2017.

Isin, E. F. and Nielsen, G. M. (Eds.): Acts of citizenship, Zed Books, London, New York, 2008.

Kitchin, R. M.: Towards geographies of cyberspace, Prog. Hum. Geogr., 22, 385-406, https://doi.org/10.1191/030913298668331585, 2016. 
Marchart, O.: Die politische Differenz. Zum Denken des Politischen bei Nancy, Lefort, Badiou, Laclau und Agamben, Suhrkamp, Berlin, 2011.

Massey, D.: For space. Reprint, Sage, London, 2010.

McKinnon, C.: Toward a feminist theory of the state, Harward University Press, Cambridge, MA, 1989.

Mouffe, C.: Agonistics. Thinking the world politically, Verso, New York, 2013.

Mustafa, D. and Brown, K. E.: The Taliban, public space, and terror in Pakistan, Erasian Geogr. Econ., 51, 496-512, https://doi.org/10.2747/1539-7216.51.4.496, 2010.

Mustafa, D., Brown, K. E., and Tillotson, M.: Antipode to terror: Spaces of performative politics, Antipode, 45, 1110-1127, https://doi.org/10.1111/anti.12014, 2013.

Oakeshott, M.: Political education (An inaugural lecture delivered at the London School of Economics and Political Sciences on March 6, 1951), in: Rationalism in politics and other essays, New and expanded, edited by: Oakeshott, M., Liberty Fund, Indianapolis, 219-238, 1991.

Petermann, S.: Rituale machen Räume. Zum kollektiven Gedenken der Schlacht von Verdun und der Landung in der Normandie, Transcript (Sozialtheorie), Bielefeld, 2007.

Rancière, J.: Das Unvernehmen. Politik und Philosophie, Suhrkamp, Frankfurt am Main, 2002.

Rawls, J.: A Theory of justice, Harward University Press, Cambridge, MA, 1971.

Runkel, S. and Everts, J.: Geographien sozialer Krisen/Krisen sozialer Geographien, Geogr. Helv., 72, 475-482, https://doi.org/10.5194/gh-72-475-2017, 2017.

Schäfer, H. (Hrsg.): Praxistheorie. Ein soziologisches Forschungsprogramm, Transcript, Bielefeld, 2014.

Schäfer, S.: Climate change adaptation in South Korea. Environmental politics in the agricultural sector, Transcript, Bielefeld, 2015

Schatzki, T. R.: The site of the social. A philosophical account of the constitution of social life and change, Pennsylvania State University Press, University Park, 2002.
Schatzki, T. R.: The timespace of human activity. On performance, society, and history as indeterminate teleological events, Lexington Books, Lanham, 2010.

Schatzki, T. R.: Spaces of practices and of large social phenomena, EspacesTemps.net, Works, available at: http://www.espacestemps.net/en/articles/ spaces-of-practices-and-of-large-social-phenomena/ (last access: 14 May 2020), 2015.

Schatzki, T. R.: Social change in a material world: A prècis, in: Handbuch Praktiken und Raum: Humangeographie nach dem Practical Turn, Herausgeber: Everts, J. und Schäfer, S.: Transcript, Bielefeld, 77-92, 2019.

Schurr, C.: Performing politics, making space. A visual ethnography of political change in Ecuador, Erdkundliches Wissen 152, Steiner, Stuttgart, 2013.

Topper, K.: Arendt and Bourdieu between word and deed, Polit. Theory, 39, 352-377, https://doi.org/10.1177/0090591711400028, 2011.

Tully, J.: Democracy and civic freedom, in: Vol. 1, Public philosophy in a new key, Cambridge Univ. Press, Cambridge, 2008.

Weisser, F.: Practices, politics, performativities: Documents in the international negotiations on climate change, Polit. Geogr., 40 , 46-55, https://doi.org/10.1016/j.polgeo.2014.02.007, 2014.

Weisser, F. and Müller-Mahn, D.: No place for the political. Microgeographies of the Paris Climate Conference 2015, Antipode, 49, 802-820, https://doi.org/10.1111/anti.12290, 2017.

Wenman, M.: Agonistic democracy. Constituent power in the era of globalization, Cambridge University Press, New York, 2013.

Werlen, B.: Sozialgeographie alltäglicher Regionalisierungen, 1, Erdkundliches Wissen 116, Steiner, Stuttgart, 1995.

Woodward, K., Jones, J. P., and Marston, S. A.: The politics of autonomous space, Prog. Hum. Geogr., 36, 204-224, 2012.

Žižek, S.: The ticklish subject. The absent centre of political ontology, Verso, London, New York, 1999. 